\title{
Multicomponent Domino [4+2]/[3+2] \\ Cycloadditions of Nitroheteroaromatics: an Efficient Synthesis of Fused Nitrogenated Polycycles
}

Isabelle Chataigner* and Serge R. Piettre*

Laboratoire des Fonctions Azotées et Oxygénées Complexes, UMR CNRS 6014, IRCOFUniversité de Rouen, Rue Tesnières, F-76821 Mont Saint Aignan, France

\section{Supporting information:}

Procedures for compound 9 and 14. Analytical data for compounds 9a, 9b, 10a, 10b, 11, 12, 13, 14, 15, 17a, 17b and $18 .{ }^{1} \mathrm{H}$ and ${ }^{13} \mathrm{C}$ Spectra for compounds $9 \mathrm{a}, 9 \mathrm{~b}, 10 \mathrm{a}, 10 \mathrm{~b}, 11$, $12,13,14,15,17 \mathrm{a}, 17 \mathrm{~b}$ and 18.

Representative procedure for the domino [4+2]/[3+2] cycloadditions under high pressure activation.

Analytical data for compound 9a

Analytical data for compound $\mathbf{9 b}$

Analytical data for compound 10a

Analytical data for compound $\mathbf{1 0 b}$

Analytical data for compound $\mathbf{1 1}$

Analytical data for compound $\mathbf{1 2}$

Analytical data for compound $\mathbf{1 3}$ 
$\begin{array}{ll}\text { Representative procedure for the reduction } & \text { S7 }\end{array}$

$\begin{array}{ll}\text { Analytical data for compound } 14 & \text { S8 }\end{array}$

$\begin{array}{ll}\text { Analytical data for compound } \mathbf{1 5} & \text { S8 }\end{array}$

$\begin{array}{ll}\text { Analytical data for compound } \mathbf{1 8} & \text { S8 }\end{array}$

$\begin{array}{ll}\text { ORTEP for compound 10a } & \text { S9 }\end{array}$

$\begin{array}{ll}\text { ORTEP for compound } \mathbf{1 0 b} & \text { S9 }\end{array}$

${ }^{1} \mathrm{H}$ NMR spectrum of compound 9a $\quad \mathrm{S} 10$

${ }^{13} \mathrm{C}$ NMR spectrum of compound 9a $\quad$ S10

${ }^{1} \mathrm{H}$ NMR spectrum of compound $\mathbf{9 b} \quad \mathrm{S} 11$

${ }^{13} \mathrm{C}$ NMR spectrum of compound $9 \mathbf{b} \quad$ S11

${ }^{1} \mathrm{H}$ NMR spectrum of compound 10a $\quad \mathrm{S} 12$

${ }^{13} \mathrm{C}$ NMR spectrum of compound 10a $\quad \mathrm{S} 12$

${ }^{1} \mathrm{H}$ NMR spectrum of compound 10b $\quad \mathrm{S} 13$

${ }^{13} \mathrm{C}$ NMR spectrum of compound 10b $\quad$ S13

${ }^{1} \mathrm{H}$ NMR spectrum of compounds 11

${ }^{13} \mathrm{C}$ NMR spectrum of compounds 11

$\begin{array}{ll}{ }^{1} \mathrm{H} \text { NMR spectrum of compounds } \mathbf{1 2} & \text { S15 }\end{array}$

$\begin{array}{ll}{ }^{13} \mathrm{C} \text { NMR spectrum of compounds } \mathbf{1 2} & \mathrm{S} 15\end{array}$

${ }^{1} \mathrm{H}$ NMR spectrum of compounds $\mathbf{1 3} \quad$ S16

${ }^{13} \mathrm{C}$ NMR spectrum of compounds $13 \quad$ S16

$\begin{array}{ll}{ }^{1} \mathrm{H} \text { NMR spectrum of compound } \mathbf{1 4} & \mathrm{S} 17\end{array}$

${ }^{13} \mathrm{C}$ NMR spectrum of compound $\mathbf{1 4} \quad \mathrm{S} 17$

${ }^{1}$ H NMR spectrum of compound $15 \quad$ S18

${ }^{13} \mathrm{C}$ NMR spectrum of compound $\mathbf{1 5} \quad \mathrm{S} 18$

${ }^{1} \mathrm{H}$ NMR spectrum of compound 17a $\quad$ S19

${ }^{13} \mathrm{C}$ NMR spectrum of compound 17a $\quad$ S19

${ }^{1} \mathrm{H}$ NMR spectrum of compound $\mathbf{1 7 b} \quad$ S20

${ }^{13} \mathrm{C}$ NMR spectrum of compound 17b $\quad$ S20

${ }^{1} \mathrm{H}$ NMR spectrum of compound $\mathbf{1 8} \quad$ S21

$\begin{array}{ll}{ }^{13} \mathrm{C} \text { NMR spectrum of compound } \mathbf{1 8} & \text { S21 }\end{array}$

General. ${ }^{1} \mathrm{H}$ NMR (300 MHz) and ${ }^{13} \mathrm{C}$ NMR (75 MHz) spectra were recorded in deuterated chloroform relative to $\left(\mathrm{CH}_{3}\right)_{4} \mathrm{Si}$ and $\mathrm{CDCl}_{3}$ respectively. Chemical shifts are expressed in 
parts per million (ppm). Low and high resolution mass spectra were recorded in either EI, CI or FAB operating in positive ion mode. IR spectra were recorded as films. Hyperbaric experiments were conducted on Unipress U101 or Psika Pressure Systems Ltd. apparati.

\section{Representative procedure for the domino $[4+2] /[3+2]$ cycloadditions under high pressure activation. Cycloaddition reaction between indole 4, ethyl vinyl ether 5 a and methyl acrylate 6a.}

To a solution of 3-nitro-1-p-toluenesulfonylindole 4 (79 $\mathrm{mg}, 0.25 \mathrm{mmol})$, in dry dichloromethane $(1 \mathrm{~mL})$ at room temperature under argon, was added ethyl vinyl ether 5a $(144 \mu \mathrm{L}, 1.5 \mathrm{mmol})$ and methyl acrylate $6 \mathbf{a}(135 \mu \mathrm{L}, 1.5 \mathrm{mmol})$. The resultant mixture was transfered into a high pressure vessel and compressed at $1.2 \mathrm{GPa}$, at room temperature. After decompression, the solvent and excess alkenes were evaporated under reduced pressure. Chromatography of the residue on silica (cyclohexane/EtOAc 7:3) afforded $\mathbf{9}(99 \mathrm{mg}, 83 \%)$ as a 55:45 mixture of diastereomers.

$\left(2 R^{*}, 6 S^{*}, 7 \mathrm{a} R^{*}, 12 \mathrm{~b} R^{*}\right)-6$-ethoxy-2-methoxycarbonyl-3,5-dioxa-8-tosyl-1,2,3,5,6,7,7a,8octahydroindolizino[8-b]indole (9a). Purification of the residue by flash chromatography (cyclohexane/EtOAc 7:3). The adduct was isolated as a white solid (m.p. $103{ }^{\circ} \mathrm{C}$ ). ${ }^{1} \mathrm{H}-\mathrm{NMR} \delta$ $1.14(\mathrm{t}, J=7.2,3 \mathrm{H}), 1.41(\mathrm{dd}, J=5.7,12.8,1 \mathrm{H}), 1.94(\mathrm{ddd}, J=8.4,12.8,13.6,1 \mathrm{H}), 2.11(\mathrm{dd}$, $J=10.6,12.8,1 \mathrm{H}), 2.30(\mathrm{~s}, 3 \mathrm{H}), 2.43(\mathrm{ddd}, J=5.3,12.8,13.6,1 \mathrm{H}), 3.44(\mathrm{qd}, J=7.2,9.6$, $1 \mathrm{H}), 3.68(\mathrm{~s}, 3 \mathrm{H}), 3.82(\mathrm{qd}, J=7.2,9.6,1 \mathrm{H}), 4.11(\mathrm{dd}, J=5.3,12.8,1 \mathrm{H}), 4.88(\mathrm{dd}, J=6.6$, $8.4,1 \mathrm{H}), 5.02(\mathrm{dd}, J=5.8,10.6,1 \mathrm{H}), 7.05(\mathrm{dd} \sim \mathrm{t}, J=7.2,1 \mathrm{H}), 7.16(\mathrm{~d}, J=7.2,2 \mathrm{H}), 7.10-7.22$ $(\mathrm{m}, 1 \mathrm{H}), 7.27(\mathrm{ddd}, J=1.5,7.2,8.3,1 \mathrm{H}), 7.45(\mathrm{~d}, J=7.2,2 \mathrm{H}), 7.60(\mathrm{~d}, J=8.3,1 \mathrm{H}) .{ }^{13} \mathrm{C}-$ NMR $\delta 14.9,21.5,32.7,41.9,52.6,63.6,65.1,80.8,83.7,97.8,117.7,125.3,126.3,127.0$ (2C), 129.8 (2C), 130.1, 132.8, 134.1, 140.4, 144.6, 169.6. MS (DCI isobutane) $\mathrm{m} / \mathrm{z}$ (relative intensity) 475 (12), 429 (100). IR 1168, 1359, 1442, 1460, 1480, 1599, 1746, 2894, 2935, 2956, $2676 \mathrm{~cm}^{-1}$. Anal. Calcd for $\mathrm{C}_{23} \mathrm{H}_{26} \mathrm{~N}_{2} \mathrm{O}_{7} \mathrm{~S} \mathrm{C}, 58.21 ; \mathrm{H}, 5.52 ; \mathrm{N}, 5.90 ; \mathrm{S}, 6.76$. Found $\mathrm{C}$, 58.35; H, 5.57; N, 5.81; S, 6.73.

$\left(2 S^{*}, 6 S^{*}, 7 \mathrm{a} R^{*}, 12 \mathrm{~b} R^{*}\right)-6$-ethoxy-2-methoxycarbonyl-3,5-dioxa-8-tosyl-1,2,3,5,6,7,7a,8octahydroindolizino[8-b]indole (9b). Purification of the residue by flash chromatography (cyclohexane/EtOAc 7:3). The adduct was isolated as a white solid (m.p. $70^{\circ} \mathrm{C}$ ). ${ }^{1} \mathrm{H}-\mathrm{NMR} \delta$ 
$1.13(\mathrm{t}, J=7.2,3 \mathrm{H}), 1.39(\mathrm{dd}, J=9.0,12.8,1 \mathrm{H}), 1.94(\mathrm{ddd}, J=8.7,12.8,13.6,1 \mathrm{H}), 2.30(\mathrm{~s}$, $3 \mathrm{H}), 2.30-2.50(\mathrm{~m}, 2 \mathrm{H}), 3.43(\mathrm{qd}, J=7.2,9.6,1 \mathrm{H}), 3.72(\mathrm{~s}, 3 \mathrm{H}), 3.82(\mathrm{qd}, J=7.2,9.6,1 \mathrm{H})$, $4.14(\mathrm{dd}, J=5.3,12.8,1 \mathrm{H}), 4.72(\mathrm{dd}, J=7.2,9.0,1 \mathrm{H}), 4.84(\mathrm{dd}, J=6.8,8.7,1 \mathrm{H}), 6.95(\mathrm{~d}, J=$ $7.2,1 \mathrm{H}), 7.03(\mathrm{dd} \sim \mathrm{t}, J=7.2,1 \mathrm{H}), 7.16(\mathrm{~d}, J=7.2,2 \mathrm{H}), 7.28(\mathrm{dd}, J=7.2,8.3,1 \mathrm{H}), 7.46(\mathrm{~d}, J$ $=7.2,2 \mathrm{H}), 7.63(\mathrm{~d}, J=8.3,1 \mathrm{H}) .{ }^{13} \mathrm{C}-\mathrm{NMR} \delta 14.9,21.5,32.9,39.9,52.7,63.7,64.3,81.2$, 84.1, 98.0, 118.0, 124.4, 126.1, 127.0 (2C), 129.8 (2C), 130.4, 132.0, 134.0, 140.7, 144.6, 170.7. MS (FAB+, 3-nitrobenzylalcohol) m/z (relative intensity) 475 (13), 429 (47), 307 (100), 289 (100). IR 1169, 1359, 1478, 1599, 1742, $2978 \mathrm{~cm}^{-1}$. Anal. Calcd for $\mathrm{C}_{23} \mathrm{H}_{26} \mathrm{~N}_{2} \mathrm{O}_{7} \mathrm{~S}$ C, 58.21; H, 5.52; N, 5.90; S, 6.76. Found C, 58.36; H, 5.62; N, 5.91; S, 6.63.

$\left(2 R^{*}, \quad 6 S^{*}, \quad 7 \mathrm{a} R^{*}, \quad 12 \mathrm{~b} R^{*}\right)-6-n$ butoxy-2-methoxycarbonyl-3,5-dioxa-8-tosyl1,2,3,5,6,7,7a,8-octahydroindolizino[8-b]indole (10a). Purification of the residue by flash chromatography (cyclohexane/EtOAc 7:3). The adduct was isolated as a white solid (m.p. $\left.141{ }^{\circ} \mathrm{C}\right) .{ }^{1} \mathrm{H}-\mathrm{NMR} \delta 0.82(\mathrm{t}, J=7.2,3 \mathrm{H}), 1.15-1.60(\mathrm{~m}, 5 \mathrm{H}), 1.94(\mathrm{ddd}, J=8.3,12.8,13.6$, $1 \mathrm{H}), 2.11(\mathrm{dd}, J=10.2,12.6,1 \mathrm{H}), 2.31(\mathrm{~s}, 3 \mathrm{H}), 2.43(\mathrm{ddd}, J=5.3,6.6,13.6,1 \mathrm{H}), 3.36(\mathrm{qd}, J=$ $6.8,9.4,1 \mathrm{H}), 3.69(\mathrm{~s}, 3 \mathrm{H}), 3.76(\mathrm{qd}, J=6.8,9.4,1 \mathrm{H}), 4.10(\mathrm{dd}, J=5.3,12.8,1 \mathrm{H}), 4.86(\mathrm{dd}, J$ $=6.4,8.3,1 \mathrm{H}), 5.02(\mathrm{dd}, J=5.7,10.2,1 \mathrm{H}), 6.90-7.52(\mathrm{~m}, 3 \mathrm{H}), 7.16(\mathrm{~d}, J=8.3,2 \mathrm{H}), 7.44(\mathrm{~d}$, $J=8.3,2 \mathrm{H}), 7.59(\mathrm{~d}, J=7.9,1 \mathrm{H}) .{ }^{13} \mathrm{C}-\mathrm{NMR} \delta 13.8,19.1,21.5,31.4,32.7,41.9,52.6,65.2$, 68.1, 80.9, 83.7, 98.1, 117.8, 125.4, 126.3, 127.0 (2C), 129.8 (2C), 130.1, 132.9, 134.2, 140.5, 144.6, 169.6. MS (DCI isobutane) $\mathrm{m} / \mathrm{z}$ (relative intensity) 503 (21), 429 (100). IR 1167, 1359, 1482, 1596, 1750, 2872, 2934, $2955 \mathrm{~cm}^{-1}$. Anal. Calcd for $\mathrm{C}_{25} \mathrm{H}_{30} \mathrm{~N}_{2} \mathrm{O}_{7} \mathrm{~S} \mathrm{C}, 59.75 ; \mathrm{H}, 6.02 ; \mathrm{N}$, 5.57; S, 6.38. Found C, 59.84; H, 6.03; N, 5.55; S, 6.39.

$\left(2 S^{*}, 6 S^{*}, 7 \mathrm{a} R^{*}, 12 \mathrm{~b} R^{*}\right)-6-n b u t o x y-2-m e t h o x y c a r b o n y l-3,5-d i o x a-8-t o s y l-1,2,3,5,6,7,7 \mathrm{a}, 8-$ octahydroindolizino[8- $b$ ]indole (10b). Purification of the residue by flash chromatography (cyclohexane/EtOAc 7:3). The adduct was isolated as a white solid (m.p. $119^{\circ} \mathrm{C}$ ). ${ }^{1} \mathrm{H}-\mathrm{NMR} \delta$ $0.82(\mathrm{t}, J=7.2,3 \mathrm{H}), 1.15-1.70(\mathrm{~m}, 5 \mathrm{H}), 1.97(\mathrm{ddd}, J=8.7,12.8,13.6,1 \mathrm{H}), 2.31(\mathrm{~s}, 3 \mathrm{H}), 2.36$ $(\mathrm{dd}, J=7.5,12.8,1 \mathrm{H}), 2.44(\mathrm{ddd}, J=5.3,6.8,13.6,1 \mathrm{H}), 3.36(\mathrm{qd}, J=6.8,9.8,1 \mathrm{H}), 3.72(\mathrm{~s}$, $3 \mathrm{H}), 3.76(\mathrm{qd}, J=6.8,9.8,1 \mathrm{H}), 4.13(\mathrm{dd}, J=5.3,12.8,1 \mathrm{H}), 4.73(\mathrm{dd}, J=7.5,9.0,1 \mathrm{H}), 4.83$ $(\mathrm{dd}, J=6.8,8.7,1 \mathrm{H}), 6.95(\mathrm{~d}, J=7.2,1 \mathrm{H}), 7.03(\mathrm{dd} \approx \mathrm{t}, J=7.2,7.2,1 \mathrm{H}), 7.16(\mathrm{~d}, J=8.3,2 \mathrm{H})$, $7.28(\mathrm{dd}, J=7.2,7.9,1 \mathrm{H}), 7.46(\mathrm{~d}, J=8.3,2 \mathrm{H}), 7.63(\mathrm{~d}, J=7.9,1 \mathrm{H}) .{ }^{13} \mathrm{C}-\mathrm{NMR} \delta 13.8,19.1$, 21.5, 31.4, 32.9, 39.9, 52.7, 64.4, 68.1, 81.3, 84.1, 98.3, 118.0, 124.5, 126.1, 127.0 (2C), 129.8 (2C), 130.4, 132.1, 134.0, 140.7, 144.6, 170.7. MS (DCI isobutane) m/z (relative intensity) 503 (18), 429 (100). IR 1165, 1359, 1478, 1599, 1738, 2872, 2930, $2957 \mathrm{~cm}^{-1}$. 
Anal. Calcd for $\mathrm{C}_{25} \mathrm{H}_{30} \mathrm{~N}_{2} \mathrm{O}_{7} \mathrm{~S} \mathrm{C}, 59.75 ; \mathrm{H}, 6.02 ; \mathrm{N}, 5.57 ; \mathrm{S}, 6.38$. Found C, 59.88; H, 6.24; N, $5.35 ; \mathrm{S}, 6.02$.

\section{2-tbutyloxycarbonyl-6-ethoxy-3,5-dioxa-8-tosyl-1,2,3,5,6,7,7a,8-octahydroindolizino[8-}

b]indole (11). Purification of the residue by flash chromatography (cyclohexane/EtOAc 8:2). The adduct was isolated as a white solid as a mixture of diastereomers. Major diastereomer (endo : $\left.2 S^{*}, 6 S^{*}, 7 \mathrm{a} R^{*}, 12 \mathrm{~b} R^{*}\right){ }^{1} \mathrm{H}-\mathrm{NMR} \delta 1.15(\mathrm{t}, J=7.2,3 \mathrm{H}), 1.20-1.45(\mathrm{~m}, 1 \mathrm{H}), 1.36(\mathrm{~s}$, $9 \mathrm{H}), 1.85-2.15(\mathrm{~m}, 1 \mathrm{H}), 2.25-2.50(\mathrm{~m}, 2 \mathrm{H}), 2.30(\mathrm{~s}, 3 \mathrm{H}), 3.35-3.52(\mathrm{~m}, 1 \mathrm{H}), 3.73-3.88(\mathrm{~m}$, $1 \mathrm{H}), 4.08(\mathrm{dd}, J=5.3,13.2,1 \mathrm{H}), 4.62(\mathrm{dd}, J=6.8,8.9,1 \mathrm{H}), 4.80-4.92(\mathrm{~m}, 1 \mathrm{H}), 6.90-7.70(\mathrm{~m}$, $8 \mathrm{H}) .{ }^{13} \mathrm{C}-\mathrm{NMR} \delta 14.9,21.5,27.8$ (3C), 32.6, 41.8, 63.6, 65.2, 81.6, 82.6, 83.5, 97.8, 117.9, 125.5, 126.3, 127.1 (2C), 129.7 (2C), 130.1, 133.1, 134.1, 140.5, 144.5, 168.2. Minor diastereomer (exo: $\left.2 R^{*}, 6 S^{*}, 7 \mathrm{a} R^{*}, 12 \mathrm{~b} R^{*}\right){ }^{1} \mathrm{H}-\mathrm{NMR} \delta 1.13(\mathrm{t}, J=7.2,3 \mathrm{H}), 1.20-1.45(\mathrm{~m}$, $1 \mathrm{H}), 1.41(\mathrm{~s}, 9 \mathrm{H}), 1.85-2.15(\mathrm{~m}, 1 \mathrm{H}), 2.25-2.50(\mathrm{~m}, 2 \mathrm{H}), 2.30(\mathrm{~s}, 3 \mathrm{H}), 3.35-3.52(\mathrm{~m}, 1 \mathrm{H})$, $3.73-3.88(\mathrm{~m}, 1 \mathrm{H}), 4.15(\mathrm{dd}, J=5.3,12.8,1 \mathrm{H}), 4.80-4.92(\mathrm{~m}, 2 \mathrm{H}), 6.90-7.70(\mathrm{~m}, 8 \mathrm{H}) .{ }^{13} \mathrm{C}-$ NMR $\delta$ 15.0, 21.5, 27.9 (3C), 32.9, 39.8, 63.6, 64.5, 82.0, 82.2, 84.2, 98.0, 118.1, 124.5, 126.1, 127.0 (2C), 129.7 (2C), 130.3, 132.5, 134.0, 140.7, 144.5, 169.4. MS (DCI isobutane) $\mathrm{m} / \mathrm{z}$ (relative intensity) 518(12), 517 (50), 471 (100). IR 1170, 1360, 1478, 1599, 1732, 2979 $\mathrm{cm}^{-1}$. Anal. Calcd for $\mathrm{C}_{26} \mathrm{H}_{32} \mathrm{~N}_{2} \mathrm{O}_{7} \mathrm{~S} \mathrm{C}, 60.45 ; \mathrm{H}, 6.24 ; \mathrm{N}, 5.42 ; \mathrm{S}, 6.21$. Found C, 60.54; H, $6.44 ; \mathrm{N}, 5.41 ; \mathrm{S}, 6.12$.

\section{6-ethoxy-3,5-dioxa-2-phenoxycarbonyl-8-tosyl-1,2,3,5,6,7,7a,8-octahydroindolizino[8-}

b]indole (12). Purification of the residue by flash chromatography (cyclohexane/EtOAc 8:2). The adduct was isolated as a white solid as a mixture of diastereomers. Major diastereomer (endo: $\left.2 S^{*}, 6 S^{*}, 7 \mathrm{a} R^{*}, 12 \mathrm{~b} R^{*}\right){ }^{1} \mathrm{H}-\mathrm{NMR} \delta 1.16(\mathrm{t}, J=7.2,3 \mathrm{H}), 1.42-1.56(\mathrm{~m}, 1 \mathrm{H}), 1.90-2.13$ $(\mathrm{m}, 1 \mathrm{H}), 2.35-2.55(\mathrm{~m}, 2 \mathrm{H}), 2.30(\mathrm{~s}, 3 \mathrm{H}), 3.35-3.55(\mathrm{~m}, 1 \mathrm{H}), 3.75-3.95(\mathrm{~m}, 1 \mathrm{H}), 4.18(\mathrm{dd}, J=$ $5.6,12.8,1 \mathrm{H}), 4.91(\mathrm{dd}, J=6.4,8.3,1 \mathrm{H}), 4.98(\mathrm{dd}, J=6.2,9.2,1 \mathrm{H}), 6.90-7.70(\mathrm{~m}, 13 \mathrm{H}) .{ }^{13} \mathrm{C}-$ NMR $\delta$ 15.0, 21.6, 32.9, 40.1, 63.8, 64.5, 81.4, 84.4, 98.2, 118.1, 121.0 (2C), 124.4, 126.2, 126.2, 127.1 (2C), 129.5 (2C), 129.8 (2C), 130.5, 132.3, 134.0, 140.8, 144.7, 150.5, 169.2. Minor diastereomer (exo: $\left.2 R^{*}, 6 S^{*}, 7 \mathrm{a} R^{*}, 12 \mathrm{~b} R^{*}\right){ }^{1} \mathrm{H}-\mathrm{NMR} \delta 1.16(\mathrm{t}, J=7.2,3 \mathrm{H}), 1.90-2.13$ (m, 1H), 2.15-2.35 (m, 2H), $2.27(\mathrm{~s}, 3 \mathrm{H}), 2.35-2.55(\mathrm{~m}, 1 \mathrm{H}), 3.35-3.55(\mathrm{~m}, 1 \mathrm{H}), 3.75-3.95(\mathrm{~m}$, $1 \mathrm{H}), 4.00-4.25(\mathrm{~m}, 1 \mathrm{H}), 4.80-5.05(\mathrm{~m}, 1 \mathrm{H}), 5.24(\mathrm{dd}, J=5.3,10.5,1 \mathrm{H}), 6.90-7.70(\mathrm{~m}, 13 \mathrm{H})$. ${ }^{13} \mathrm{C}-\mathrm{NMR} \delta 15.0,21.5,32.7,42.0,63.7,65.2,80.8,83.7,98.0,118.1,120.9$ (2C), 125.3, 126.5, 126.6, 127.1 (2C), 129.6 (2C), 129.8 (2C), 130.3, 132.8, 134.1, 140.6, 144.7, 149.8, 167.8. MS (FAB+) m/z (relative intensity) 538 (9), 537 (22), 491 (100), 460 (49). IR 1169, 
1359, 1478, 1491, 1597, 1759, 2894, 2930, 2977, 3009, $3043 \mathrm{~cm}^{-1}$. Anal. Calcd for $\mathrm{C}_{28} \mathrm{H}_{28} \mathrm{~N}_{2} \mathrm{O}_{7} \mathrm{~S} \mathrm{C}, 62.67 ; \mathrm{H}, 5.26 ; \mathrm{N}, 5.22 ; \mathrm{S}, 5.98$. Found C, 62.58; H, 4.91; N, 5.17; S, 6.62.

\section{6-ethoxy-2-(naphtalen-1-yloxycarbonyl)-3,5-dioxa-8-tosyl-1,2,3,5,6,7,7a,8-}

octahydroindolizino[8- $b$ ]indole (13). Purification of the residue by flash chromatography (cyclohexane/EtOAc 8:2). The adduct was isolated as a white solid as a mixture of diastereomers. Major diastereomer (endo: $\left.2 S^{*}, 6 S^{*}, 7 \mathrm{a} R^{*}, 12 \mathrm{~b} R^{*}\right){ }^{1} \mathrm{H}-\mathrm{NMR} \delta 1.16(\mathrm{t}, J=7.2$, $3 \mathrm{H}), 1.45-1.60(\mathrm{~m}, 1 \mathrm{H}), 1.90-2.20(\mathrm{~m}, 1 \mathrm{H}), 2.27(\mathrm{~s}, 3 \mathrm{H}), 2.40-2.60(\mathrm{~m}, 2 \mathrm{H}), 3.40-3.60(\mathrm{~m}$, $1 \mathrm{H}), 3.80-4.00(\mathrm{~m}, 1 \mathrm{H}), 4.22(\mathrm{dd}, J=5.3,12.8,1 \mathrm{H}), 4.95(\mathrm{dd}, J=6.4,8.3,1 \mathrm{H}), 5.16(\mathrm{dd}, J=$ $6.8,9.4,1 \mathrm{H}), 7.03-7.78(\mathrm{~m}, 12 \mathrm{H}), 7.82(\mathrm{~d}, J=7.9,2 \mathrm{H}), 7.96(\mathrm{~d}, J=8.3,1 \mathrm{H}) .{ }^{13} \mathrm{C}-\mathrm{NMR} \delta$ $15.0,21.5,33.0,39.9,63.8,64.5,81.7,84.5,98.3,117.5,118.2,121.3,124.4,125.2,126.2$, 126.3, 126.5, 126.6, 127.1 (2C), 127.9, 129.8 (2C), 130.5, 132.2, 132.7, 133.9, 134.6, 140.8, 144.7, 146.4, 169.1. Minor diastereomer (exo: $\left.2 R^{*}, 6 S^{*}, 7 \mathrm{a} R^{*}, 12 \mathrm{~b} R^{*}\right){ }^{1} \mathrm{H}-\mathrm{NMR} \delta 1.16(\mathrm{t}, J=$ 7.2, 3H), 1.55-1.70 (m, 1H), 1.90-2.40 (m, 2H), $2.18(\mathrm{~s}, 3 \mathrm{H}), 2.40-2.80(\mathrm{~m}, 1 \mathrm{H}), 3.40-3.60(\mathrm{~m}$, $1 \mathrm{H}), 6.80-4.00(\mathrm{~m}, 1 \mathrm{H}), 4.18(\mathrm{dd}, J=5.3,13.2,1 \mathrm{H}), 4.94(\mathrm{dd}, J=6.4,8.3,1 \mathrm{H}), 5.41(\mathrm{dd}, J=$ 5.6, 10.5, 1H), 6.95-7.85 (m, 15H). ${ }^{13} \mathrm{C}-\mathrm{NMR} \delta 15.0,21.4,32.7,42.8,63.8,65.2,81.1,84.0$, 98.1, 117.6, 118.0, 120.9, 125.3, 125.4, 126.0, 126.3, 126.5, 126.6, 127.1 (2C), 128.0, 129.8 (2C), 130.3, 132.2, 132.7, 134.0, 134.6, 140.6, 144.8, 146.4, 167.8. MS (CI+, isobutane) m/z (relative intensity) 588 (35), 587 (100), 433 (33), 399 (60). IR 1169, 1365, 1460, 1474, 1602, 1765, 2974, $3053 \mathrm{~cm}^{-1}$. Anal. Calcd for $\mathrm{C}_{32} \mathrm{H}_{30} \mathrm{~N}_{2} \mathrm{O}_{7} \mathrm{~S} \mathrm{C}, 65.51 ; \mathrm{H}, 5.15$. Found C, 65,65; H, 5,34 .

$\left(3 \mathrm{a} R^{*}, \quad 5 S^{*}, \quad 9 R^{*}, \quad 10 \mathrm{a} R^{*}\right)$-5-ethoxy-9-methoxycarbonyl-3-tosyl-3,3a,4,5,6,8,9,10octahydropyrrolo[2,3- $h$ ]indolizine (17a) Purification of the residue by flash chromatography (cyclohexane/EtOAc 75:25). The adduct was isolated as a white solid (m.p. $\left.136^{\circ} \mathrm{C}\right) .{ }^{1} \mathrm{H}-\mathrm{NMR} \delta 1.16(\mathrm{t}, J=7.2,3 \mathrm{H}), 1.41(\mathrm{dd}, J=4.1,12.4,1 \mathrm{H}), 1.97-2.14(\mathrm{~m}, 2 \mathrm{H}), 2.27-$ $2.42(\mathrm{~m}, 1 \mathrm{H}), 2.39$ (s, 3H), $3.44(\mathrm{qd}, J=7.2,9.4,1 \mathrm{H}), 3.66(\mathrm{~s}, 3 \mathrm{H}), 3.72(\mathrm{dd}, J=5.6,12.4$, $1 \mathrm{H}), 3.81(\mathrm{qd}, J=7.2,9.4,1 \mathrm{H}), 4.78(\mathrm{dd}, J=6.8,8.7,1 \mathrm{H}), 4.82(\mathrm{dd}, J=4.1,10.7,1 \mathrm{H}), 5.13$ $(\mathrm{d}, J=4.1,1 \mathrm{H}), 6.38(\mathrm{~d}, J=4.1,1 \mathrm{H}), 7.29(\mathrm{~d}, J=8.3,2 \mathrm{H}), 7.61(\mathrm{~d}, J=8.3,2 \mathrm{H}) .{ }^{13} \mathrm{C}-\mathrm{NMR} \delta$ 15.0, 21.6, 32.0, 40.7, 52.6, 61.5, 63.5, 80.9, 87.0, 97.8, 114.5, 127.3 (2C), 129.9 (2C), 132.0, 133.4, 144.5, 170.1. MS (DCI isobutane) m/z (relative intensity) 425 (63), 379 (100). IR: 1169, 1356, 1440, 1596, 1614, 1747, $2958 \mathrm{~cm}^{-1}$. Anal. Calcd for $\mathrm{C}_{19} \mathrm{H}_{24} \mathrm{~N}_{2} \mathrm{O}_{7} \mathrm{~S} \mathrm{C}, 53.76 ; \mathrm{H}$, 5.70; N, 6.60; S, 7.55. Found C, 53.84; H, 5.79; N, 6.57; S, 7.31. 
$\left(3 \mathrm{a} R^{*}, \quad 5 S^{*}, \quad 9 S^{*}, \quad 10 \mathrm{a} R^{*}\right)-5$-ethoxy-9-methoxycarbonyl-3-tosyl-3,3a,4,5,6,8,9,10octahydropyrrolo[2,3-h]indolizine (17b) Purification of the residue by flash chromatography (cyclohexane/EtOAc 75:25). The adduct was isolated as a white solid (m.p. $\left.57^{\circ} \mathrm{C}\right) .{ }^{1} \mathrm{H}-\mathrm{NMR} \delta 1.15(\mathrm{t}, J=7.2,3 \mathrm{H}), 1.44(\mathrm{dd}, J=9.0,12.8,1 \mathrm{H}), 2.04(\mathrm{ddd}, \mathrm{J}=8.7,12.4$, $13.6,1 \mathrm{H}), 2.17-2.40(\mathrm{~m}, 2 \mathrm{H}), 2.39(\mathrm{~s}, 3 \mathrm{H}), 3.41(\mathrm{qd}, J=7.2,9.4,1 \mathrm{H}), 3.68(\mathrm{~s}, 3 \mathrm{H}), 3.77(\mathrm{dd}, J$ $=5.6,12.4,1 \mathrm{H}), 3.80(\mathrm{qd}, J=7.2,9.4,1 \mathrm{H}), 4.58(\mathrm{dd}, J=7.5,9.0,1 \mathrm{H}), 4.75(\mathrm{dd}, J=6.4,8.7$, $1 \mathrm{H}), 5.00(\mathrm{~d}, J=4.1,1 \mathrm{H}), 6.42(\mathrm{~d}, J=4.1,1 \mathrm{H}), 7.30(\mathrm{~d}, J=8.3,2 \mathrm{H}), 7.61(\mathrm{~d}, J=8.3,2 \mathrm{H})$. ${ }^{13} \mathrm{C}-\mathrm{NMR} \delta$ 15.0, 21.7, 32.1, 39.3, 52.7, 61.2, 63.6, 81.0, 87.1, 97.9, 113.7, 127.4 (2C), 129.9 (2C), 132.7, 133.3, 144.6, 170.8. MS (DCI isobutane) m/z (relative intensity) 425 (18), 379 (100). IR 1169, 1355, 1439, 1597, 1739, $2924 \mathrm{~cm}^{-1}$. Anal. Calcd for $\mathrm{C}_{19} \mathrm{H}_{24} \mathrm{~N}_{2} \mathrm{O}_{7} \mathrm{~S} \mathrm{C}, 53.76$; H, 5.70; N, 6.60; S, 7.55. Found C, 53.62; H, 5.73; N, 6.51; S, 7.56.

\section{Representative procedure for the reduction of $9 \mathrm{a}$.}

Raney nickel was washed with methanol and introduced in a $50 \mathrm{~mL}$ flask containing $10 \mathrm{~mL}$ of methanol. $\mathrm{H}_{2}$ was streamed in the reaction vessel. A solution of tetracycle 9a $(60 \mathrm{mg}, 1.13 \mathrm{mmol})$ in methanol $(10 \mathrm{~mL})$ was added. The reaction vessel was compressed under an hydrogen pressure of 15 bars and stirred at room temperature for 48 hours. After decompression, the reaction medium was filtered on celite, washed with methanol (5 times). After evaporation of the solvent, the residue was chromatographed on silica (EtOAc) to afford 15 (47 $\mathrm{mg}, 98 \%)$ as a white solid.

$\left(2 R^{*}, 6 \mathrm{a} R^{*}, 11 \mathrm{~b} R^{*}\right)-2-h y d r o x y-7-t o s y l-1,2,5,6,6 \mathrm{a}, 7-h e x a h y d r o p y r r o l i z i n i[1-b]$ indol-3-one (14). Purification of the residue by flash chromatography (EtOAc). ${ }^{1} \mathrm{H}-\mathrm{NMR} \delta 1.74$ (dd, $J=$ $1.9,14.5,1 \mathrm{H}), 1.91(\mathrm{dd}, J=7.5,14.5,1 \mathrm{H}), 1.95-2.40(\mathrm{~m}, 3 \mathrm{H}), 2.30(\mathrm{~s}, 3 \mathrm{H}), 2.65$ (ddd, $J=$ $7.5,10,2,12.1,1 \mathrm{H}), 3.80(\mathrm{ddd}, J=2.6,7.2,12.1,1 \mathrm{H}), 4.13(\mathrm{dd}, J=3.6,6.6,1 \mathrm{H}), 4.31(\mathrm{dd}, J$ $=1.9,7.5,1 \mathrm{H}), 7.08(\mathrm{ddd} \approx \mathrm{dt}, J=0.9,7.2,7.5,1 \mathrm{H}), 7.15(\mathrm{~d}, J=7.9,2 \mathrm{H}), 7.28(\mathrm{ddd}, J=1.1$, $7.2,8.3,1 \mathrm{H}), 7.47(\mathrm{~d}, J=7.9,2 \mathrm{H}), 7.54(\mathrm{dd}, J=0.9,7.5,1 \mathrm{H}), 7.62(\mathrm{~d}, J=8.3,1 \mathrm{H}) .{ }^{13} \mathrm{C}-\mathrm{NMR}$ $\delta 21.6,36.5,40.5,41.3,70.0,73.1,76.3,116.2,125.9,125.9,127.1$ (2C), 129.7 (2C), 130.3, 132.9, 133.7, 141.6, 144.6, 174.5. MS (DCI isobutane) $\mathrm{m} / \mathrm{z}$ (relative intensity) 386 (26), 385 (100), 231 (85). IR 1168, 1353, 1462, 1684, 2925, 2961, $3333 \mathrm{~cm}^{-1}$. Anal. Calcd for $\mathrm{C}_{20} \mathrm{H}_{20} \mathrm{~N}_{2} \mathrm{O}_{4} \mathrm{~S}$ C, 62.48; H, 5.24; N, 7.29; S, 8.34. Found C, 62.28; H, 4.88; N, 6.99; S, 7.91. 


\section{$\left(2 S^{*}, 6 \mathrm{a} R^{*}, 11 \mathrm{~b} R^{*}\right)-2$-hydroxy-7-tosyl-1,2,5,6,6a,7-hexahydropyrrolizini[1-b]indol-3-one}

(15) Purification of the residue by flash chromatography (EtOAc). The adduct was isolated as a white solid (m.p. $\left.194^{\circ} \mathrm{C}\right) .{ }^{1} \mathrm{H}-\mathrm{NMR} \delta 1.76(\mathrm{dd}, J=9.8,13.2,1 \mathrm{H}), 2.20-2.45(\mathrm{~m}, 3 \mathrm{H}), 2.31$ (s, 3H), 2.67 (ddd, $J=6.8,7.5,12.1,1 \mathrm{H}), 3.64$ (bs, 1H), 3.91 (ddd, $J=1.5,8.1,12.1,1 \mathrm{H}$ ), $4.19(\mathrm{dd}, J=1.5,7.5,1 \mathrm{H}), 4.84(\mathrm{dd} \approx \mathrm{t}, J=9.8,9.8,1 \mathrm{H}), 7.02(\mathrm{dd} \approx \mathrm{t}, J=7.5,7.5,1 \mathrm{H}), 7.06$ $(\mathrm{dd} \approx \mathrm{t}, J=7.5,7.5,1 \mathrm{H}), 7.16(\mathrm{~d}, J=8.3,2 \mathrm{H}), 7.29(\mathrm{ddd}, J=1.5,7.5,8.3,1 \mathrm{H}), 7.51(\mathrm{~d}, J=$ 8.3, 2H), $7.66(\mathrm{~d}, J=8.3,1 \mathrm{H}) .{ }^{13} \mathrm{C}-\mathrm{NMR} \delta 21.6,35.0,40.5,43.8,70.8,71.3,73.1,116.4$, 123.9, 125.6, 127.1 (2C), 129.8 (2C), 130.4, 132.6, 133.5, 141.8, 144.7, 174.5. MS (DCI isobutane) $\mathrm{m} / \mathrm{z}$ (relative intensity) 386 (25), 385 (100), 231 (20). IR 1169, 1356, 1463, 1599, $1694,3350 \mathrm{~cm}^{-1}$. Anal. Calcd for $\mathrm{C}_{20} \mathrm{H}_{20} \mathrm{~N}_{2} \mathrm{O}_{4} \mathrm{~S} \mathrm{C}, 62.48 ; \mathrm{H}, 5.24 ; \mathrm{N}, 7.29 ; \mathrm{S}, 8.34$. Found C, 62.35; H, 5.39; N, 7.26; S, 8.22.

\section{$\left(3 \mathrm{a} R^{*}, \quad 8 S^{*}, \quad 9 \mathrm{a} S^{*}\right)-8$-hydroxy-3-tosyloctahydropyrrolo[2,3-h]pyrrolizin-7-one}

Purification of the residue by flash chromatography (EtOAc). The adduct was isolated as a white solid (m.p. $194^{\circ} \mathrm{C}$ ). ${ }^{1} \mathrm{H}-\mathrm{NMR} \delta 1.58-1.78$ (m, 2H), 1.82-2.12 (m, 2H), $2.24(\mathrm{dd}, J=7.2$, $12.1,1 \mathrm{H}), 2.32(\mathrm{dd}, J=6.2,13.7,1 \mathrm{H}), 2.39(\mathrm{~s}, 3 \mathrm{H}), 2.88-3.02(\mathrm{~m}, 1 \mathrm{H}), 3.10(\mathrm{ddd} \approx \mathrm{td}, J=7.2$, $7.2,10.9,1 \mathrm{H}), 3.42(\mathrm{ddd} \approx \mathrm{td}, J=6.4,6.4,10.9,1 \mathrm{H}), 3.61(\mathrm{~d}, J=6.4,1 \mathrm{H}), 3.86(\mathrm{dd}, J=7.5$, $12.1,1 \mathrm{H}), 4.12$ (bs, 1H), 4.50 (dd, $J=7.2,8.7,1 \mathrm{H}), 7.29$ (d, $J=8.3,2 \mathrm{H}), 7.63$ (d, $J=8.3$, 2H). ${ }^{13} \mathrm{C}-\mathrm{NMR} \delta 21.5,33.9,36.6,42.0,43.1,49.0,67.9,71.2,72.3,127.5$ (2C), 129.9 (2C), 133.3, 144.2, 175.6. MS (DCI isobutane) m/z (relative intensity) 338 (20), 337 (100). IR 1160, 1344, 1433, 1597, 1693, 2894, 2945, 2976, $3367 \mathrm{~cm}^{-1}$. Anal. Calcd for $\mathrm{C}_{16} \mathrm{H}_{20} \mathrm{~N}_{2} \mathrm{O}_{4} \mathrm{~S}$ C, 57.12; H, 5.99; N, 8.33; S, 9.53. Found C, 57.08; H, 6.03; N, 8.44; S, 9.49.

\section{ORTEP for compound 10a}




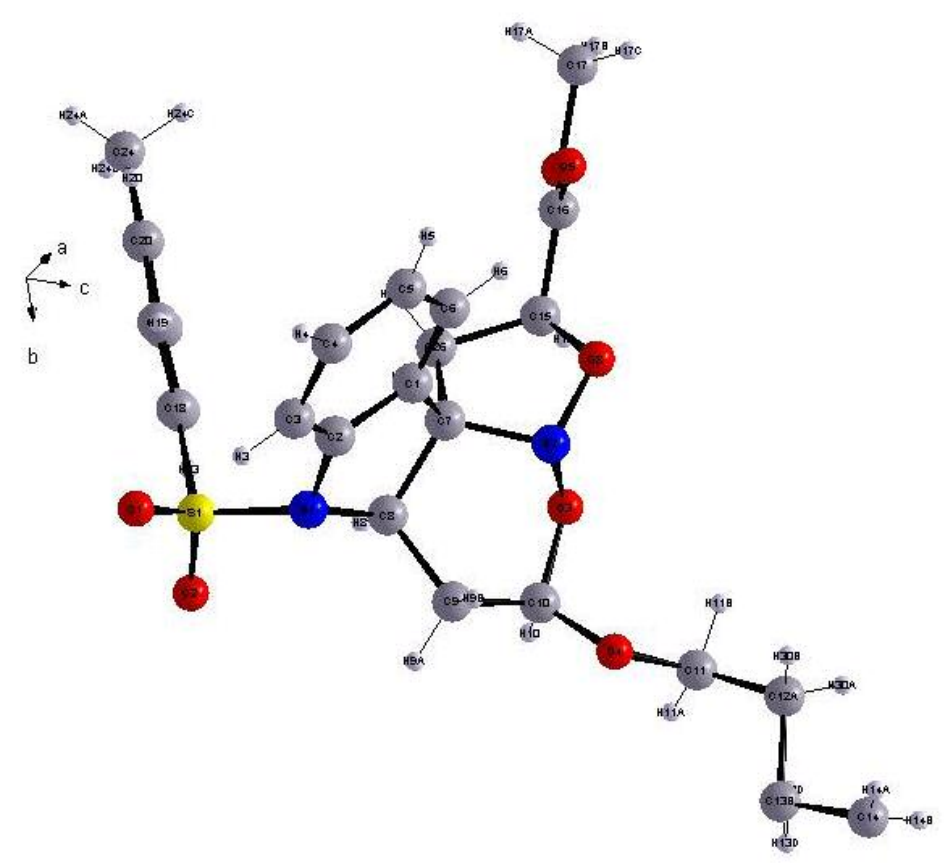

ORTEP for compound $10 b$

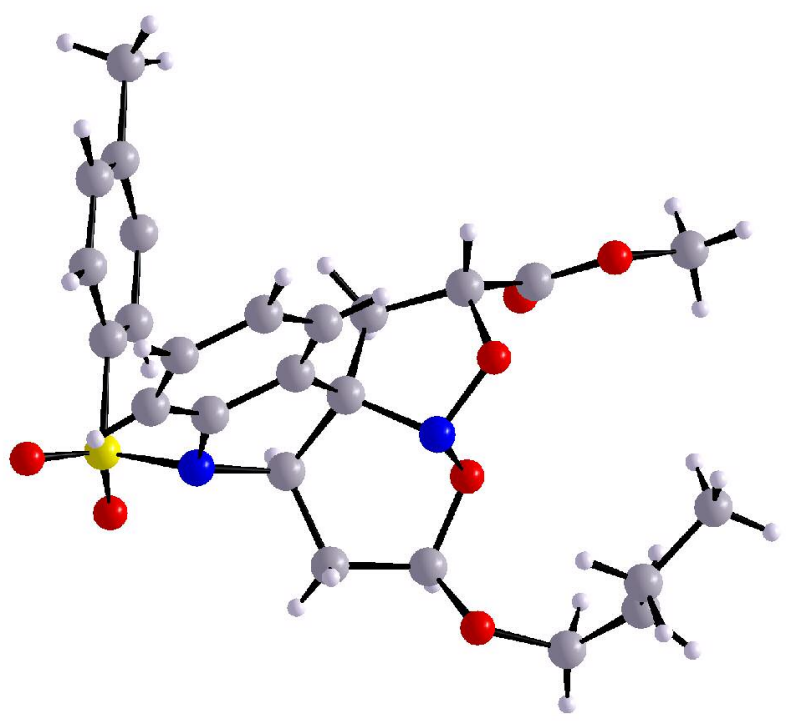

Sq 


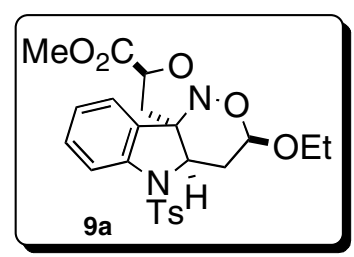

Un ind dullow
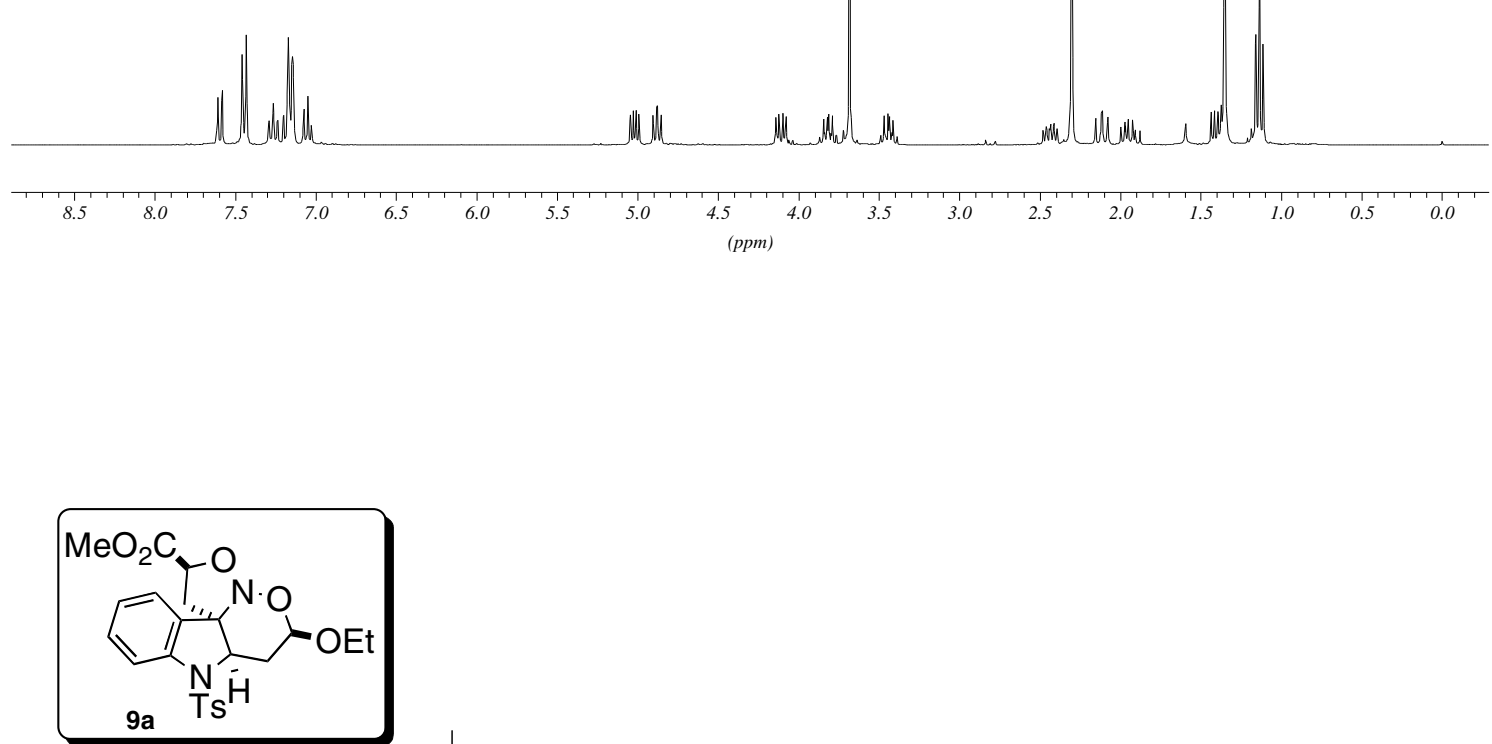

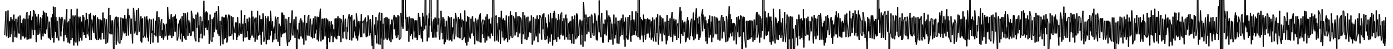

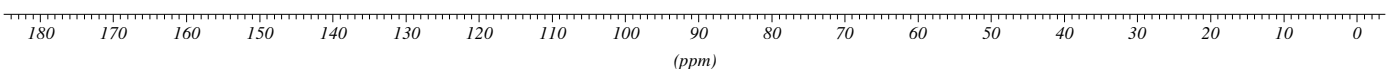



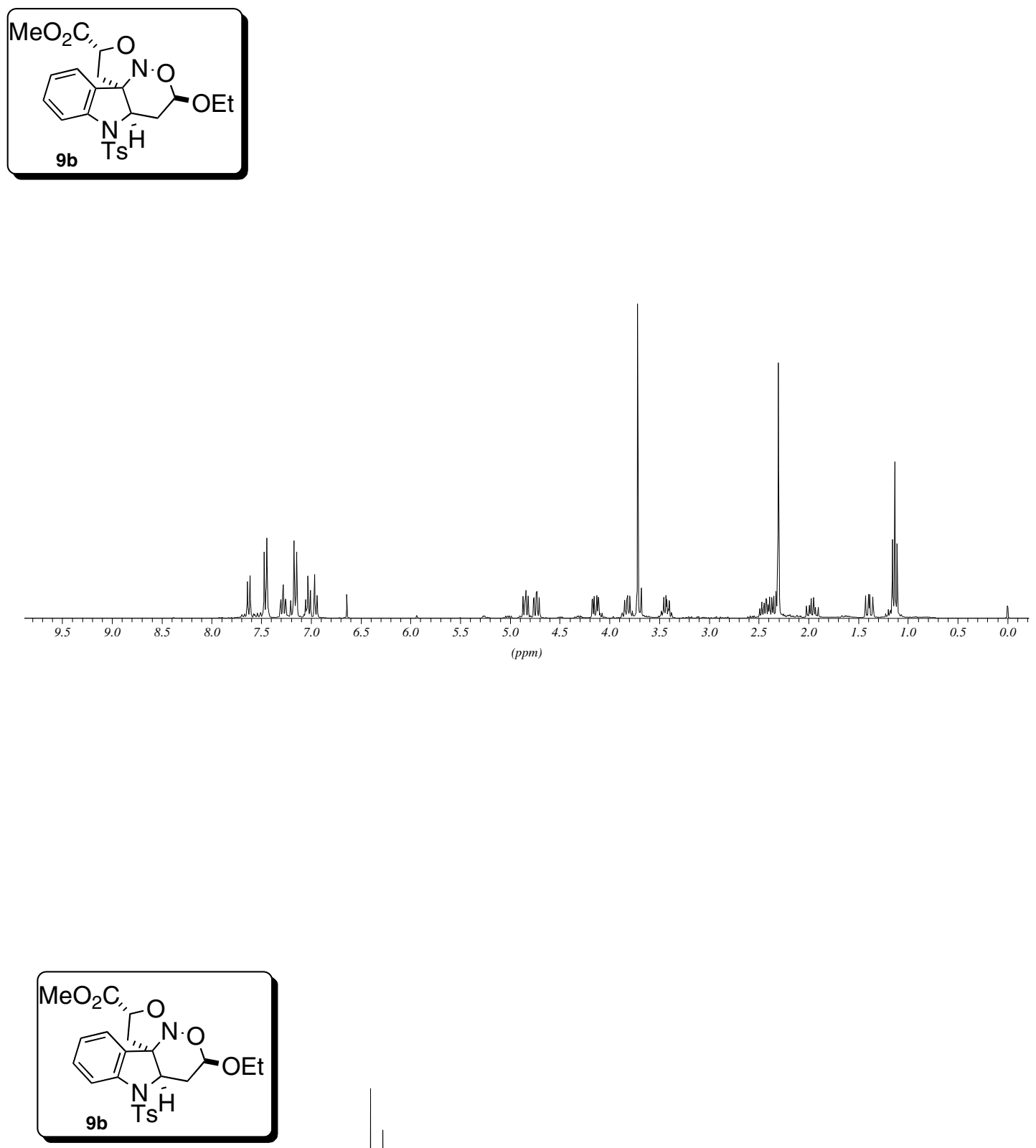

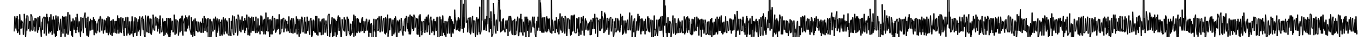

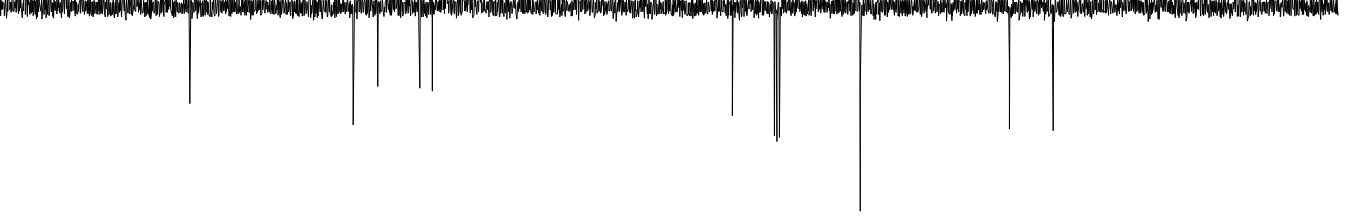

180
100 

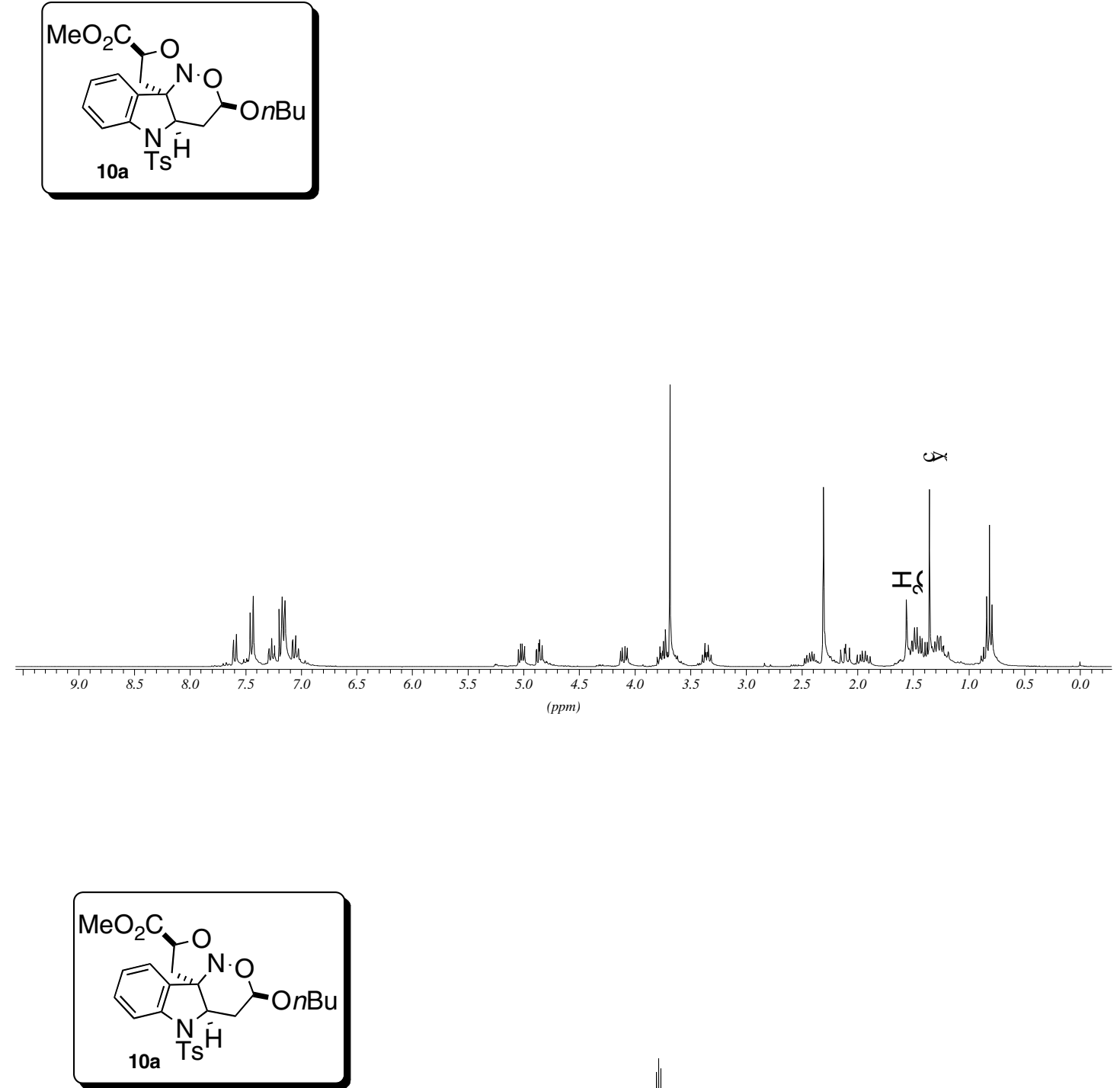

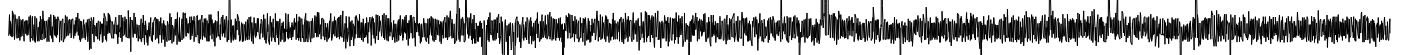

200
180



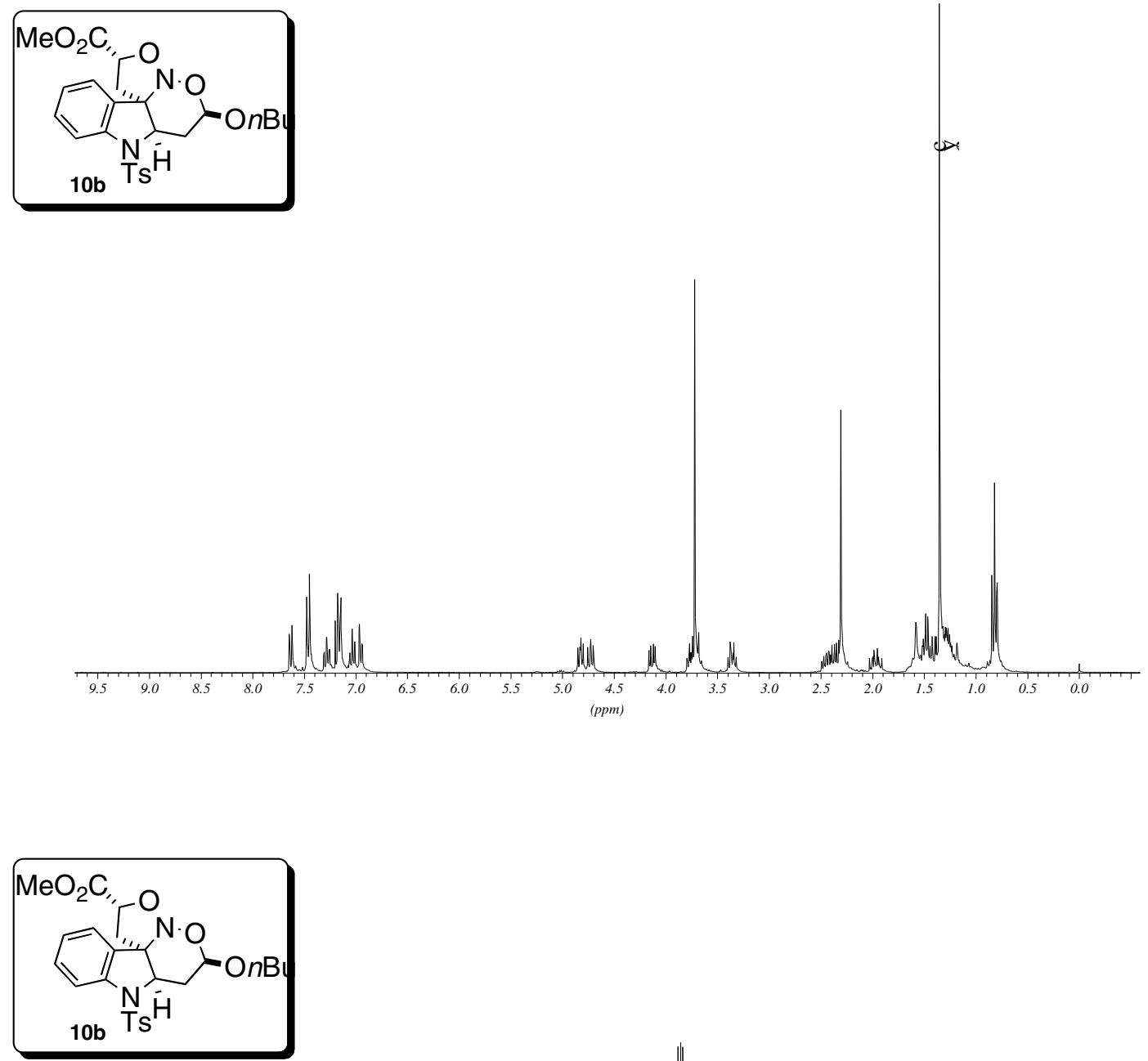

Whw

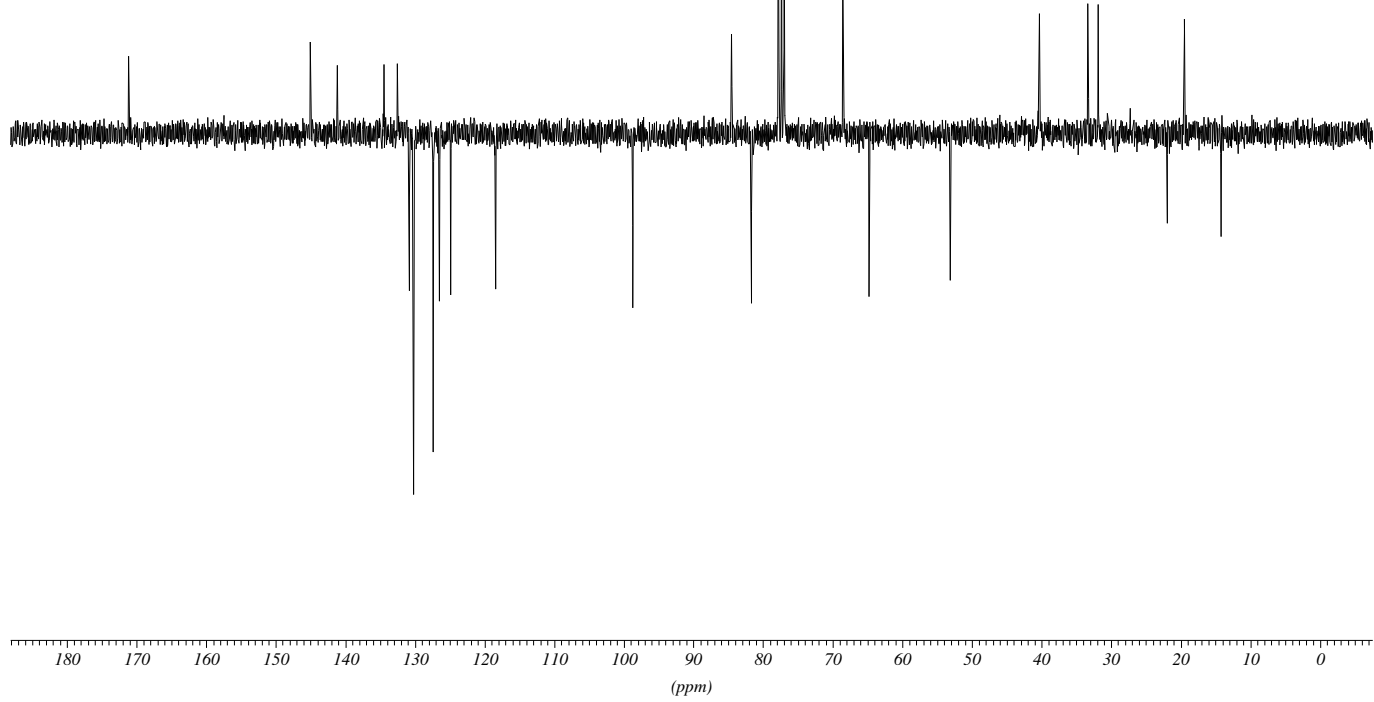



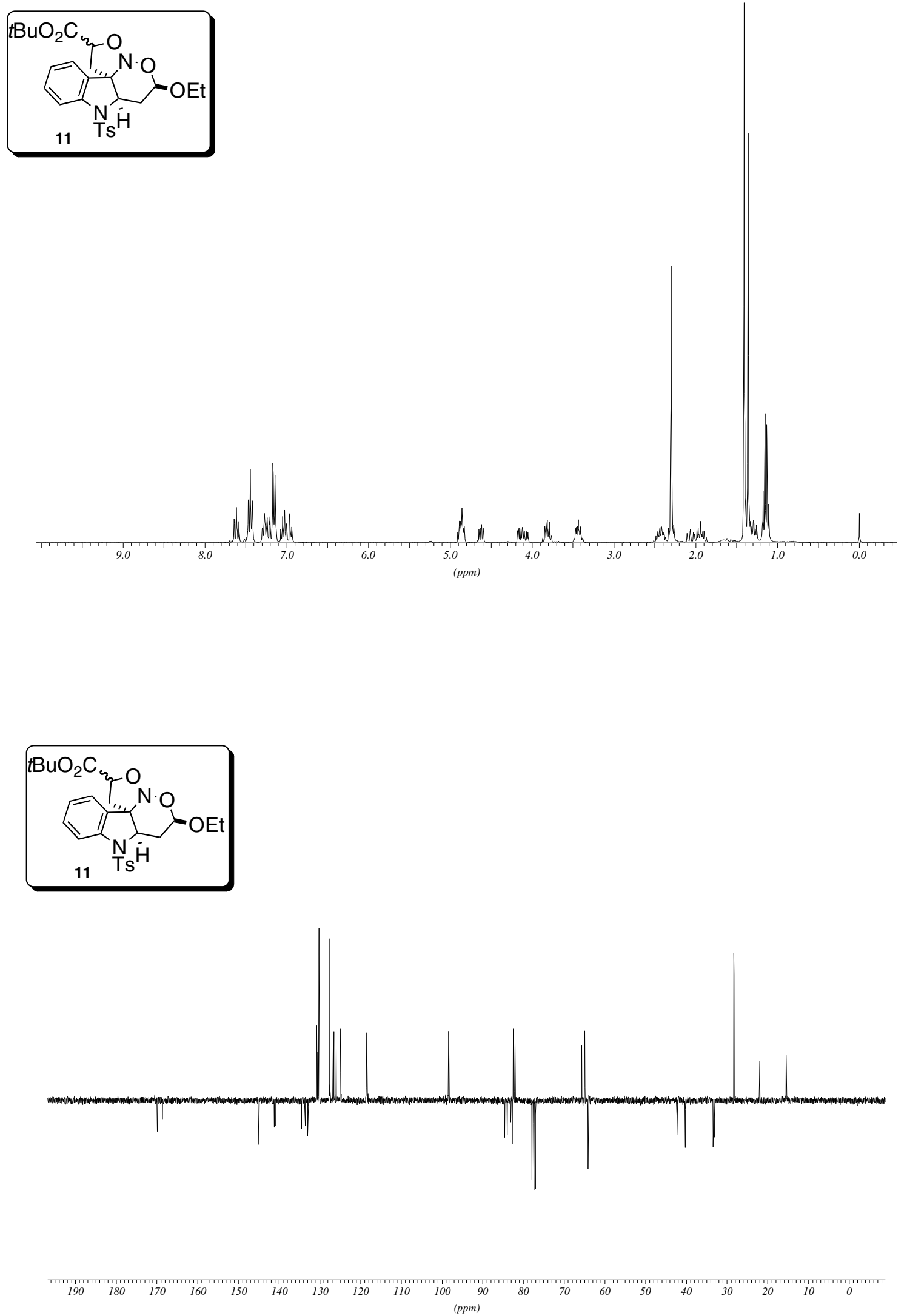

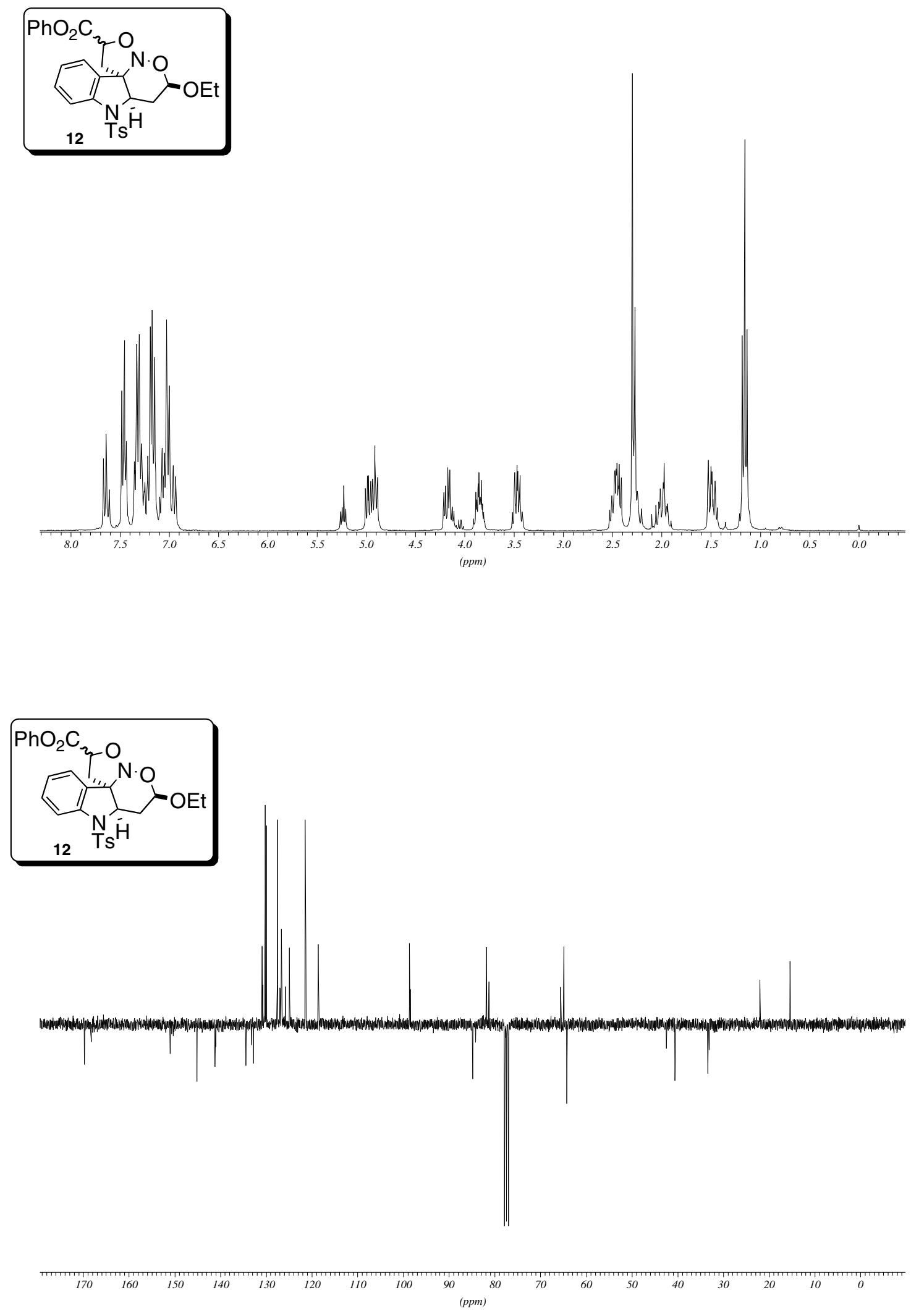

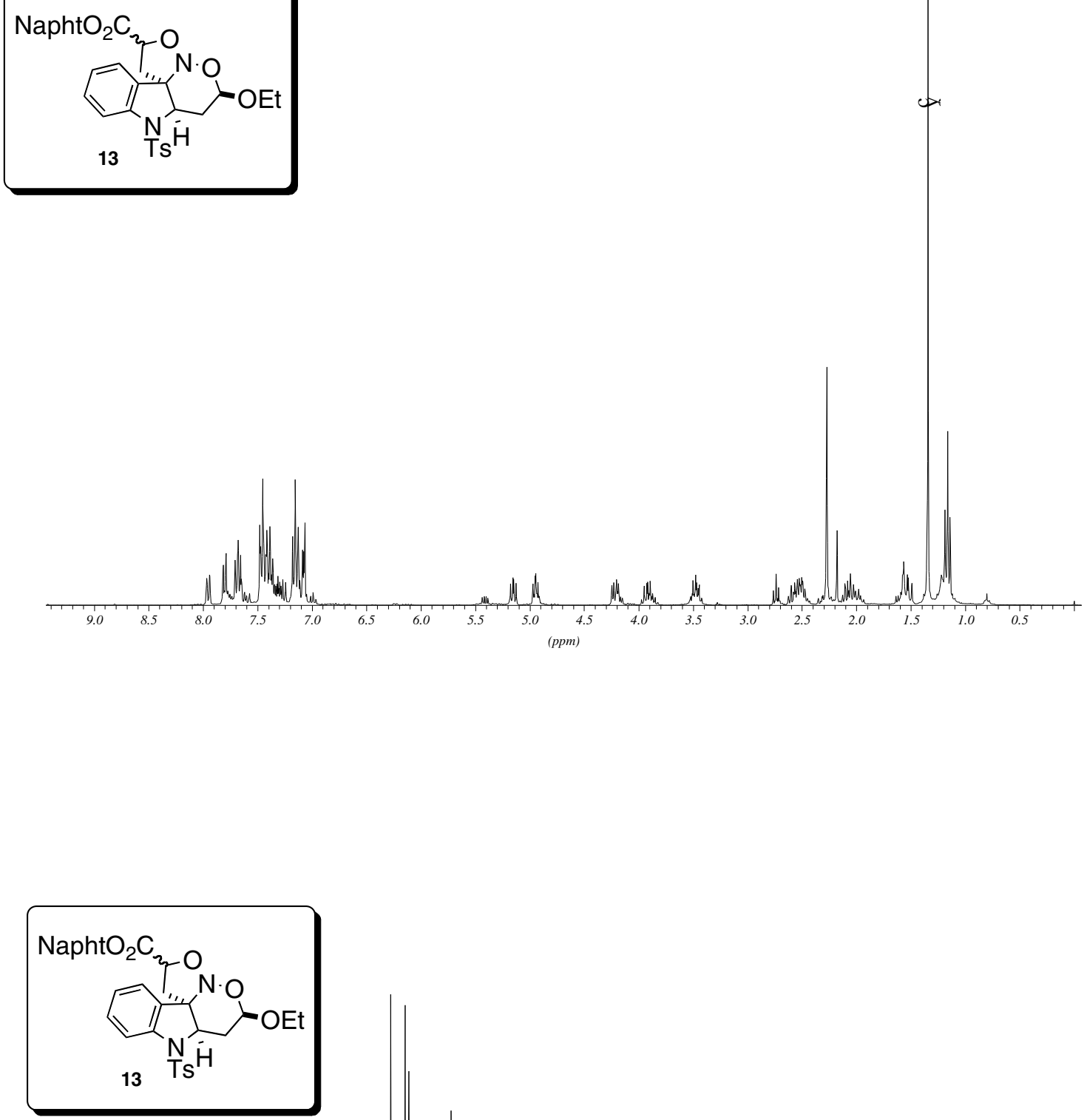

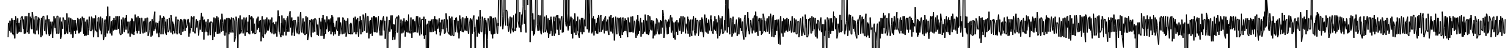
$\left.1\right|^{1}$

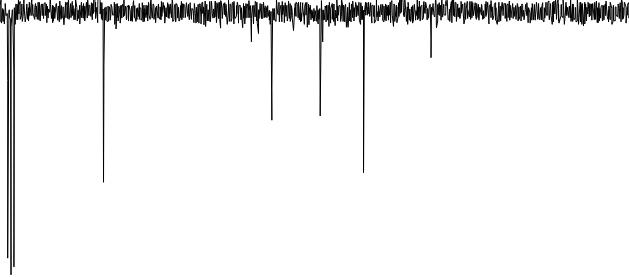



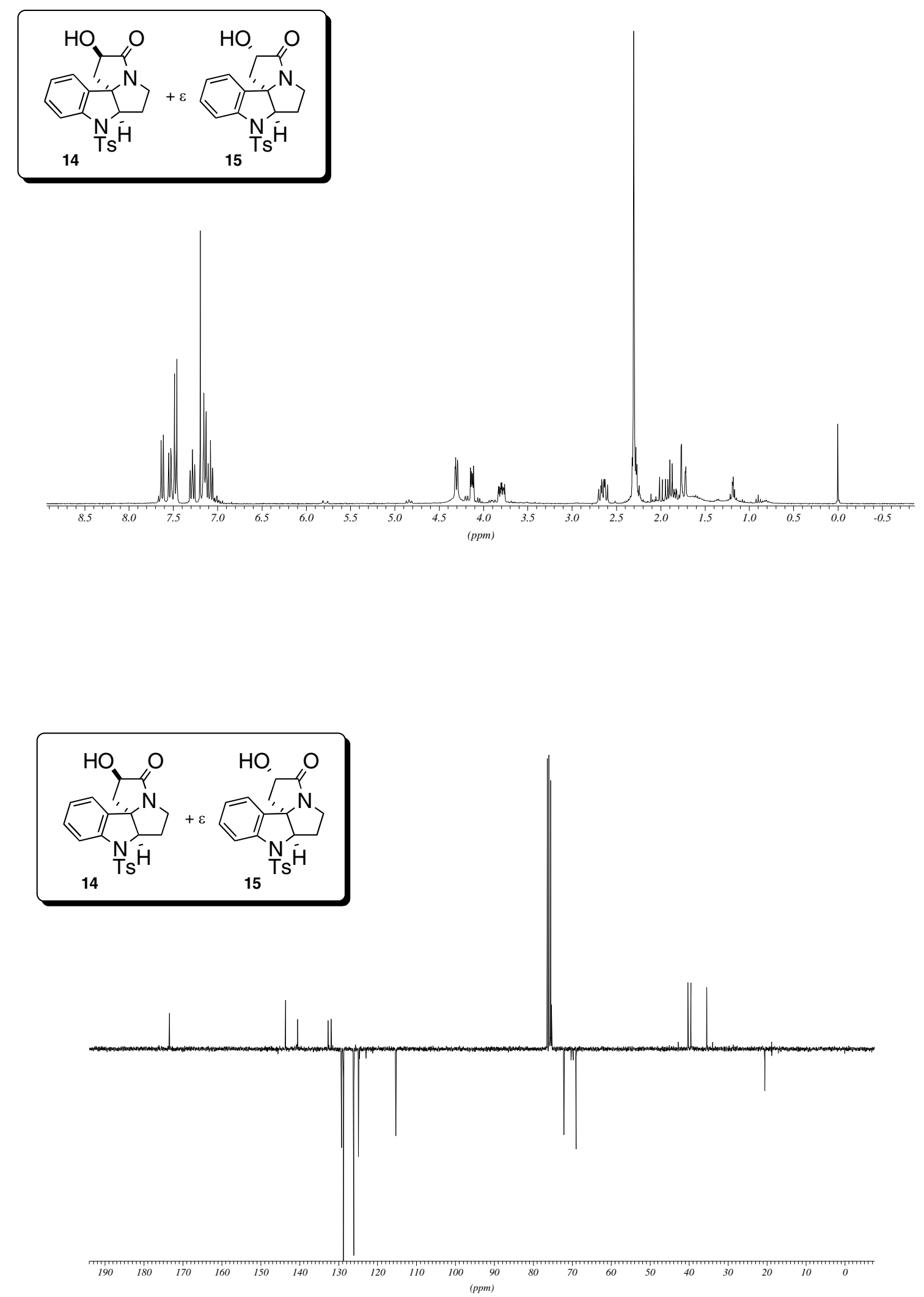

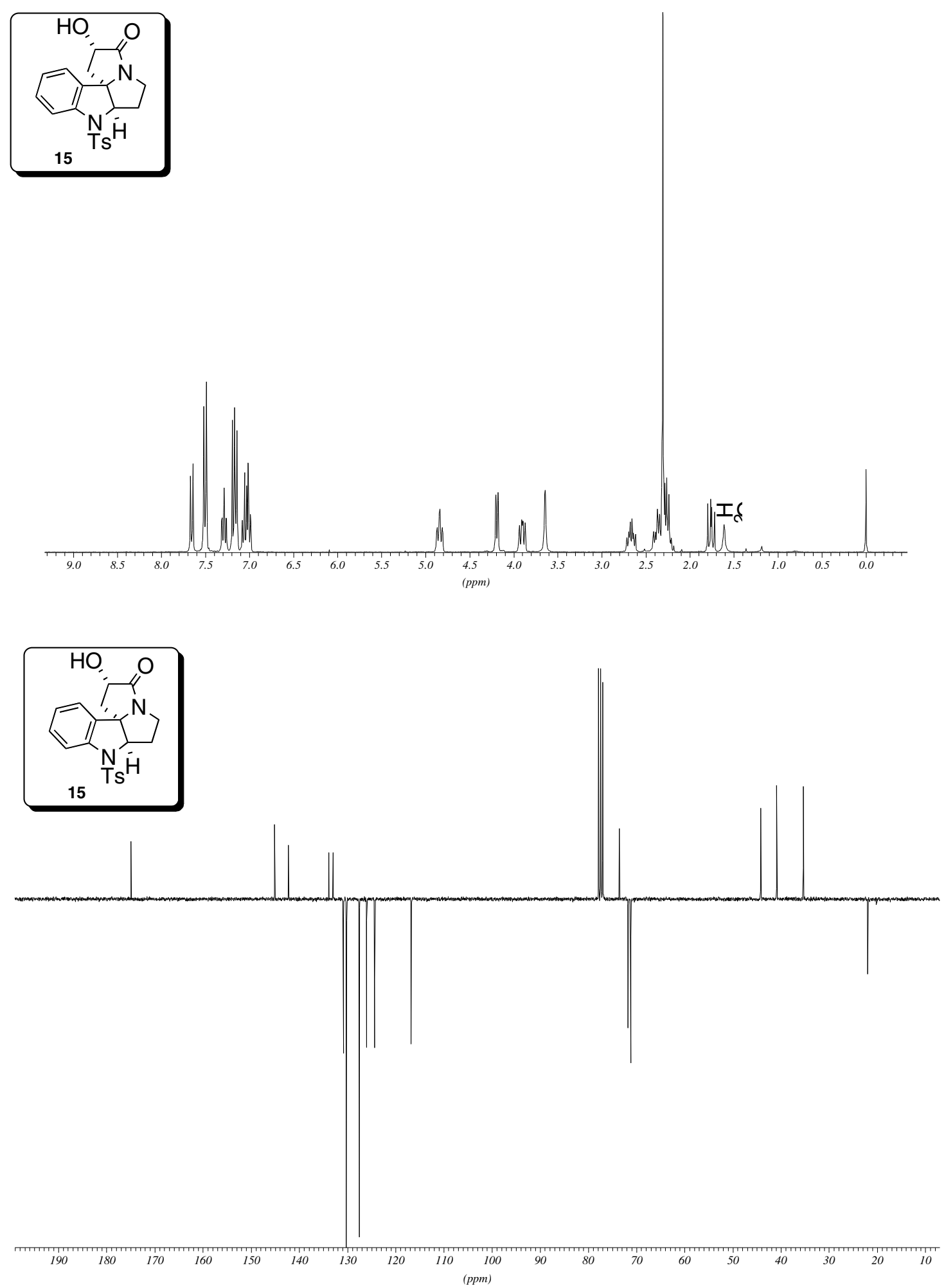

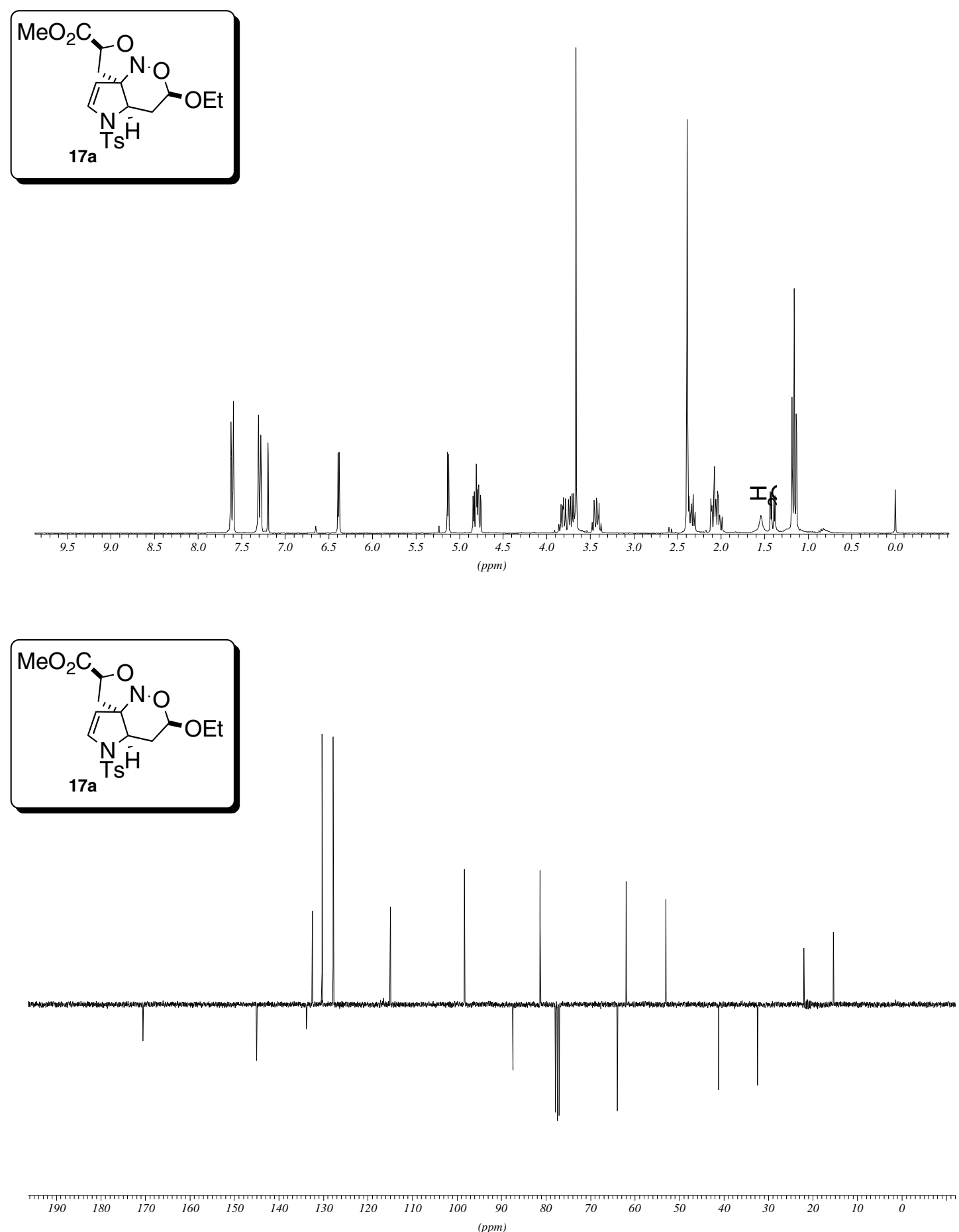

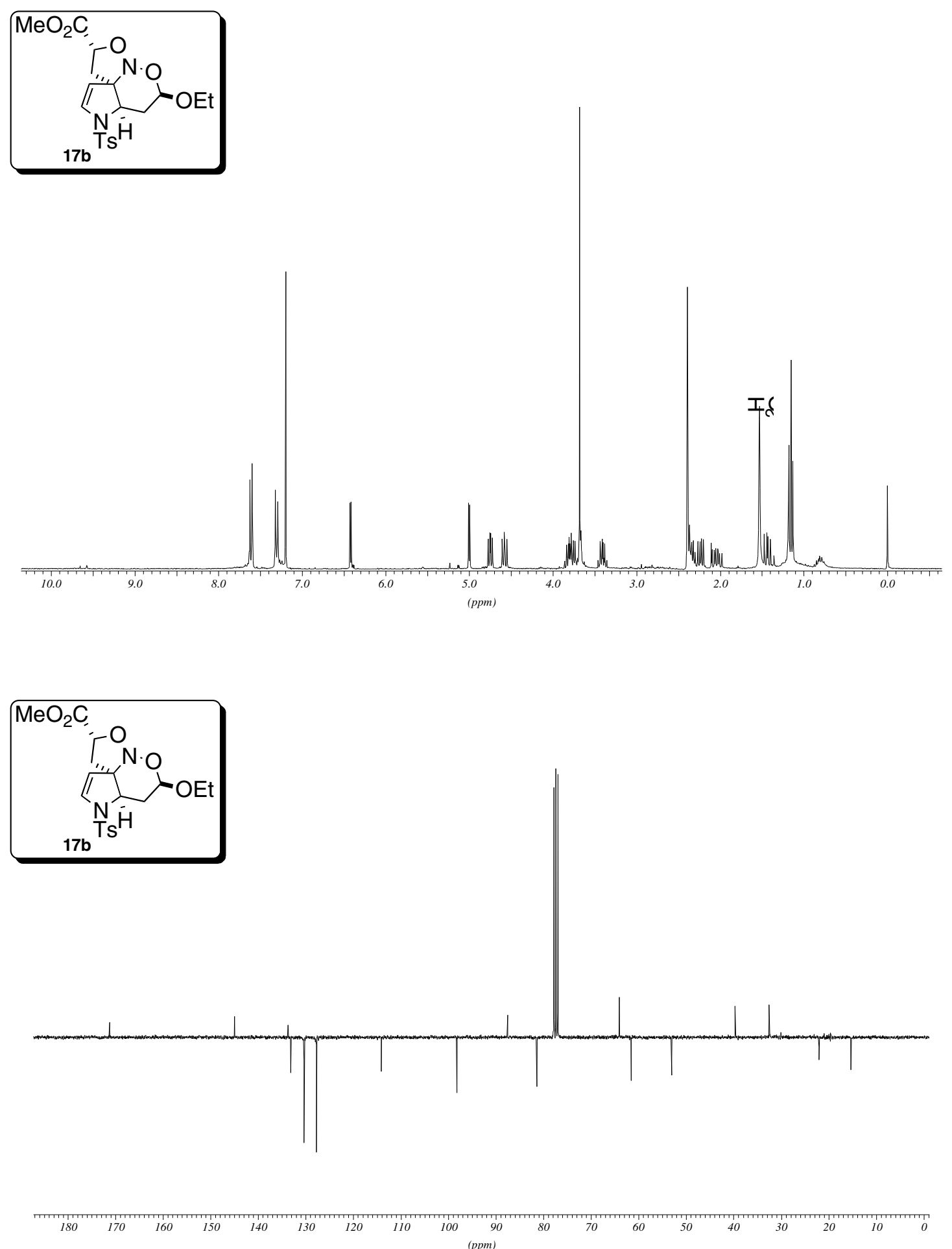

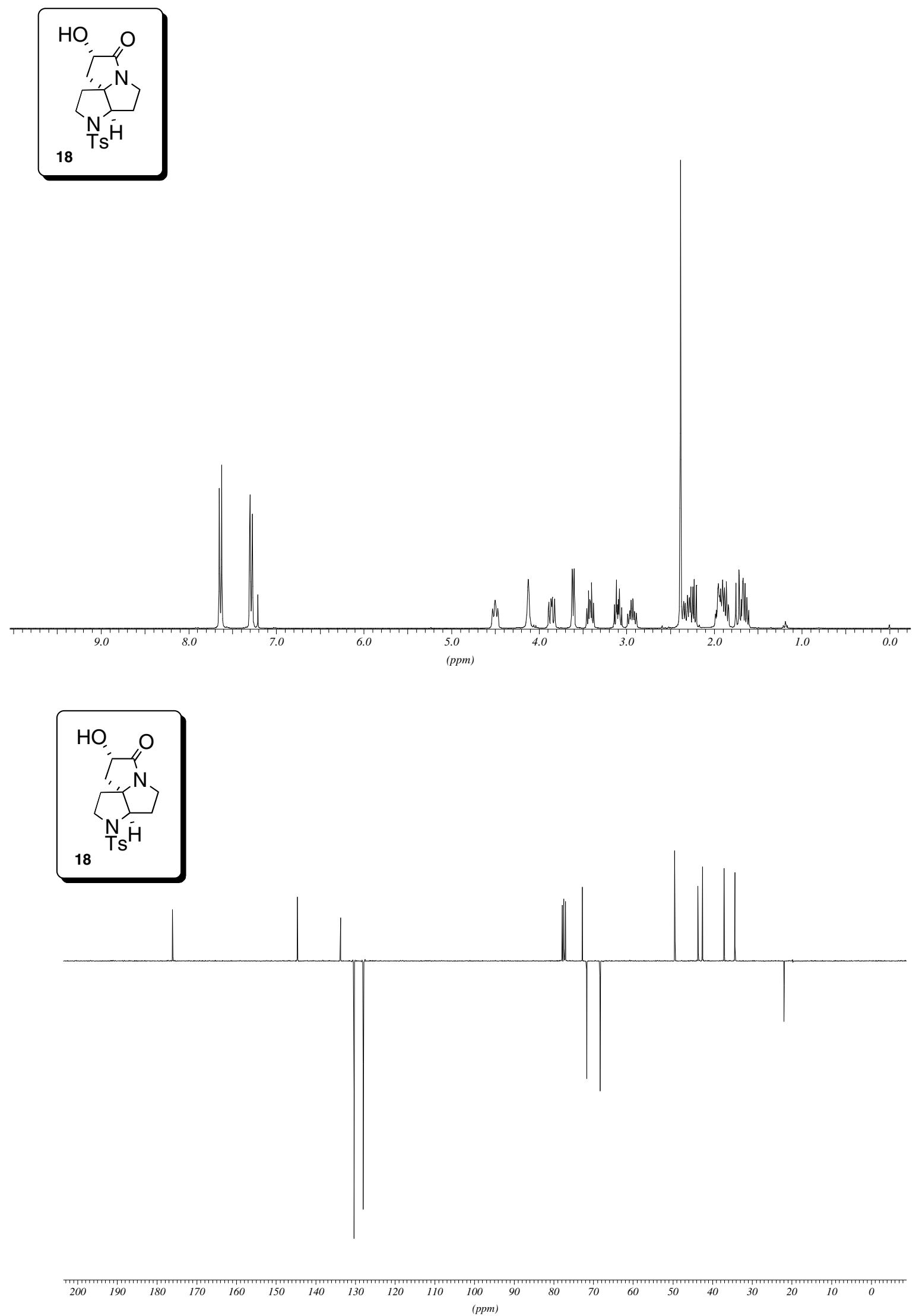